\title{
Singular Cauchy problem for a certain linear and 2nd order equation
}

\author{
In memory of professor Nobuhisa Iwasaki
}

By

\author{
Jiichiroh URABE
}

\begin{abstract}
In this paper we consider the structure, in particular the singularities, of solutions of singular Cauchy problem for the following operator $L$ with holomorphic coefficients in the neighbourhood of the origin of $C^{2}$ under some conditions

$$
L=D_{t}^{2}-\left(x+b t^{2}\right) D_{x}^{2}-a(t, x) D_{t}-c(t, x) D_{x}-d(t, x) .
$$

We construct its solution by so-called asymptotic expansion method and study its structure by the monodromy theory of the hypergeometric function.
\end{abstract}

\section{Introduction and Results}

We consider non-characteristic Cauchy problem with singular Cauchy data for a linear partial differential equation with holomorphic coefficients in the complex domain.

This problem has been investigated by Y. Hamada, J. Leray, C. Wagschal [H.L.W.1], G. Nakamura [H.N.1], D. Shiltz, J. Vaillant [S.V.W.1], T. Kobayashi [Ko.1], J. Perrson [P.1], E. Leichtnam [L.1] and many authors for various cases. We can find detail in the bibliography of the book by B. Sternin \& V. Shatalov [S.S.1]. This problem for the most general operator is difficult. In this paper we treat a limited class of operators that are related to the radially symmetric operator and Gauss's hypergeometric functions, whose monodromy theory will clarify the ramification around the characteristic surfaces and the singularities, the structure of the solution of our problem.

We consider singular Cauchy problem for the following operator $L$ in the neighbourhood $\Omega$ of the origin of $C^{2}$ with the coordinate $(t, x)$.

$$
L=D_{t}^{2}-\left(x+b t^{2}\right) D_{x}^{2}-a(t, x) D_{t}-c(t, x) D_{x}-d(t, x)
$$

where $a(t, x), c(t, x)$ and $d(t, x)$ are holomorphic functions in $\Omega$. We impose the next condition $(c)$ on the coefficient $c(t, x)$ 
Condition (c): $c(0, x)=c$ where $c$ is a constant.

So we can rewrite $c(t, x)=c+t \tilde{c}(t, x)$ and

$$
L=P_{c}-t \tilde{c}(t, x) D_{x}-a(t, x) D_{t}-d(t, x)
$$

where $P_{c}=D_{t}^{2}-\left(x+b t^{2}\right) D_{x}^{2}-c D_{x}$ and $b$ and $c$ are complex numbers.

Now we study the following Cauchy problem

$$
L u(t, x)=0 \text { with the initial data }\left\{\begin{array}{l}
u(0, x)=w_{1}(x) f_{\alpha}(x) \\
u_{t}(0, x)=w_{2}(x) f_{\alpha}(x)
\end{array}\right.
$$

where $w_{1}(x)$ and $w_{2}(x)$ are holomorphic functions in $\Omega \cap\{t=0\}$ and

$$
f_{\alpha}(x)=\frac{x^{\alpha}}{\Gamma(\alpha+1)} \quad \text { which are so-called wave forms. }
$$

This operator $P_{c}$ was studied by V. Guillemin and D. Schaeffer in [G.S.1]. When $b=0$, one can find it in [U.2, 3].

As for the singular Cauchy problem, we are interested in where the singularities of the solutions appear and what kinds of singularities the solutions have. The former problem is concerned with the homogeneous part of the highest order of $L$. In case of this operator, its characteristic surfaces determined by $P_{0}$ are composed of two curves $K^{ \pm}$where

$$
\begin{array}{ll}
K^{+}=\{(t, x) ; \xi=0\} ; & \xi=x-\frac{1}{4}(1+D) t^{2} \\
K^{-}=\{(t, x) ; \eta=0\} ; & \eta=x-\frac{1}{4}(1-D) t^{2} \\
D=\sqrt{1+16 b} ; & \operatorname{Re}(D) \geq 0 .
\end{array}
$$

$K^{ \pm}$are the characteristic surfaces issuing from the origin and their union we denote by $\mathrm{K}$. When $b=\frac{-1}{16}, D=\sqrt{1+16 b}=0$, that is, $\xi=\eta$. We do not treat this case $b=\frac{-1}{16}$ in this paper. The latter problem is concerned not only with the principal part, but also with the adequate lower order term, that is, $b$ and $c$ in particular in this operator.

We introduce the auxiliary functions $U_{\alpha}^{c}(t, x)$ and $V_{\alpha}^{c}(t, x)$ as the solution of the following Cauchy problem respectively

$$
\begin{aligned}
& P_{c} U_{\alpha}^{c}=0 \quad \text { with the initial data } U_{\alpha}^{c}(0, x)=f_{\alpha}(x) \text { and } D_{t} U_{\alpha}(0, x)=0 \\
& P_{c} V_{\alpha}^{c}=0 \quad \text { with the initial data } V_{\alpha}^{c}(0, x)=0 \text { and } D_{t} V_{\alpha}^{c}(0, x)=f_{\alpha}(x) .
\end{aligned}
$$

We remark that the next explicit representations of $U_{x}(t, x)$ and $V_{x}(t, x)$ are able to be verified.

$$
\begin{aligned}
& U_{\alpha}^{c}(t, x)=\frac{\xi^{\alpha}}{\Gamma(\alpha+1)} F\left(-\alpha, \frac{1}{4}\left(1+\frac{1}{D}\right)+\frac{\alpha+c-1}{D}, \frac{1}{2}, z\right) \\
& V_{\alpha}^{c}(t, x)=\frac{t \xi^{\alpha}}{\Gamma(\alpha+1)} F\left(-\alpha, \frac{3}{4}\left(1+\frac{1}{D}\right)+\frac{\alpha+c-1}{D}, \frac{3}{2}, z\right) \quad \text { where } z=1-\frac{\eta}{\xi} .
\end{aligned}
$$


We suppose some condition on complex parameters $\alpha, b, c$. We put $Q_{1+}=$ $\{q \in Q: q \geq 1\}$ where $Q$ is the set of all rational numbers.

Condition (b): $\quad D=\sqrt{1+16 b} \notin Q_{1+}$ and $D \neq 0\left(b \neq \frac{-1}{16}\right)$.

We denote the set of all integers by $Z$.

Condition $(\alpha, b, c):. \quad \alpha+\frac{1}{4} \pm \frac{1}{D}\left(\alpha-\frac{3}{4}+c\right) \notin Z$ and

$$
\alpha+\frac{3}{4} \pm \frac{1}{D}\left(\alpha-\frac{1}{4}+c\right) \notin Z
$$

Theorem. Under Condition (b), (c) and ( $\alpha, \mathrm{b}, \mathrm{c}$.$) , in the sufficiently small$ neighbourhood $\omega$ of the origin of $C^{2}$, this Cauchy problem has a unique holomorphic solution on the universal covering space $\tilde{R}$ over $\omega-\mathrm{K}$. More precisely speaking, the solution is expressed by

$$
\begin{aligned}
u(t, x)= & \sum_{r=0}^{\infty} \sum_{k=0}^{r}\left\{u_{r, k}(t, x) U_{\alpha+r-1}^{c-k+1}(t, x)+g_{r, k}(t, x) D_{t} U_{\alpha+r}^{c-k}(t, x)\right. \\
& \left.+v_{r, k}(t, x) V_{\alpha+r-1}^{c-k+1}(t, x)+h_{r, k}(t, x) D_{t} V_{\alpha+r}^{c-k}(t, x)\right\}
\end{aligned}
$$

where $u_{r, k}, g_{r, k}, v_{r, k}$ and $h_{r, k}$ are holomorphic functions on $\omega$.

Remark. When Cauchy data has pole on the origin in the initial surface, we also obtain a unique holomorphic solution on $\tilde{R}$ by the derivation of $u(t, x)$ with respect to $\alpha$ and restriction of $\alpha$ on the integer. In this case, Condition $(\alpha, b, c)$ is reduced to the following Condition $(b, c)$,

Condition $(b, c): \quad c \notin D\left(Z \pm \frac{1}{4}\right)+\left(Z \pm \frac{1}{4}\right)$.

Corollary. Under Condition (b) and Condition (b, c.), in the sufficiently small neighbourhood $\omega$ of the origin of $C^{2}$, this Cauchy problem $L u(t, x)=0$ with initial data $u(0, x)=w_{1}(x) k_{-l}(x)$ and $u_{t}(0, x)=w_{2}(x) k_{-l}(x)$ has a unique holomorphic solution on the universal covering space $\tilde{R}$ over $\omega-\mathrm{K}$. More precisely speaking the solution is expressed by

$$
\begin{aligned}
u(t, x)= & \sum_{r=-l}^{\infty} \sum_{k=0}^{r}\left\{u_{r, k}(t, x) X_{r-1}^{c-k+1}(t, x)+\tilde{u}_{r, k}(t, x) U_{r-1}^{c-k+1}(t, x)\right. \\
& +g_{r, k}(t, x) D_{t} X_{r-1}^{c-k+1}(t, x)+\tilde{g}_{r, k}(t, x) D_{t} U_{r-1}^{c-k+1}(t, x) \\
& +v_{r, k}(t, x) Y_{r-1}^{c-k+1}(t, x)+\tilde{v}_{r, k}(t, x) V_{r-1}^{c-k+1}(t, x) \\
& \left.+h_{r, k}(t, x) D_{t} Y_{r-1}^{c-k+1}(t, x)+\tilde{h}_{r, k}(t, x) D_{t} V_{r-1}^{c-k+1}(t, x)\right\}
\end{aligned}
$$

where $u_{r, k}, g_{r, k}, v_{r, k}, h_{r, k}$ and $\tilde{u}_{r, k}, \tilde{g}_{r, k}, \tilde{v}_{r, k}, \tilde{h}_{r, k}$ are holomorphic functions on $\omega$ and $l$ is the highest degree of the poles of the initial data and

$$
X_{\alpha}^{c}(t, x)=D_{\alpha} U_{\alpha}^{c}(t, x), \quad Y_{\alpha}^{c}(t, x)=D_{\alpha} V_{\alpha}^{c}(t, x), \quad k_{\alpha}(t, x)=D_{\alpha} f_{\alpha}(t, x) .
$$

We note $X_{x}^{c}(t, x)$ and $Y_{x}^{c}(t, x)$ satisfy the Cauchy problem $P_{c} X_{x}^{c}(t, x)=0$ with 
the initial data $X_{\alpha}^{c}(0, x)=k_{\alpha}(x)$ and $D_{t} X_{\alpha}^{c}(0, x)=0$ and $P_{c} Y_{\alpha}^{c}(t, x)=0$ with the initial data $Y_{\alpha}^{c}(0, x)=0$ and $D_{t} Y_{\alpha}^{c}(0, x)=k_{\alpha}(x)$ respectively, and $k_{\alpha}(x)=$ $|\alpha+1| !(-1)^{\alpha-1} x^{\alpha}$ for $\alpha=-1,-2, \ldots$

Note that without Condition (b) and $(\alpha, b, c)$ we can treat this problem. In this case so-called degenerate case we need something else. Here we only propose one example in Appendix.

For the proof of this theorem, we construct the formal solution of this Cauchy problem in the above expansion form and then confirm the convergence of the formal solution. This theorem shows that the singularities of the solution are reduced to the singularities of the auxiliary functions which are to be studied in Appendix in detail. To construct the formal solution, first we are to prepare some calculations and properties of the operator and auxiliary functions with which we start in the next section.

\section{Auxiliary Functions}

To prove our theorem, we need the simple relations among these auxiliary functions $U_{\alpha}^{c}(t, x)$ and $V_{\alpha}^{c}(t, x)$ and their derivatives, which we describe now and their precise properties about singularities and ramifications are to be found in Appendix.

Proposition 1. Let $r, k$ be integers non-negative.

(1) $D_{x} f_{\alpha}(x)=f_{\alpha-1}(x), \quad x f_{\alpha}(x)=(\alpha+1) f_{\alpha+1}(x), \quad D_{x} k_{\alpha}(x)=k_{\alpha-1}(x)$.

(2) $P_{c}\left(D_{t} U_{\alpha+r}^{c-k}\right)=2 b t U_{\alpha+r-2}^{c-k+2}-k D_{t} U_{\alpha+r-1}^{c-k+1}, \quad P_{c}\left(D_{t} V_{\alpha+r}^{c-k}\right)=2 b t V_{\alpha+r-2}^{c-k+2}-k D_{t} V_{\alpha+r-1}^{c-k+1}$.

(3) $P_{c}\left(D_{x} U_{x}^{c}\right)=D_{x}^{2} U_{\alpha}^{c}, \quad P_{c}\left(D_{x} V_{x}^{c}\right)=D_{x}^{2} V_{x}^{c}$.

(4) $P_{c}\left(t U_{x}^{c}\right)=2 D_{t} U_{x}^{c}, \quad P_{c}\left(t V_{x}^{c}\right)=2 D_{t} V_{x}^{c}$.

(5) $P_{c}\left(x U_{x}^{c}\right)=-c U_{x}^{c}-2\left(x+b t^{2}\right) D_{x} U_{x}^{c}, \quad P_{c}\left(x V_{x}^{c}\right)=-c V_{\alpha}^{c}-2\left(x+b t^{2}\right) D_{x} V_{x}^{c}$.

(6) $P_{c}\left(t D_{t} U_{\alpha}^{c}\right)=2 D_{t}^{2} U_{\alpha}^{c}+2 b t^{2} D_{x}^{2} U_{\alpha}^{c}, \quad P_{c}\left(t D_{t} V_{\alpha}^{c}\right)=2 D_{t}^{2} V_{\alpha}^{c}+2 b t^{2} D_{x}^{2} V_{\alpha}^{c}$.

(7) $P_{c}\left(x D_{x} U_{\alpha}^{c}\right)=-c t D_{x} U_{\alpha}^{c}-\left(x+2 b t^{2}\right) D_{x}^{2} U_{\alpha}^{c}, \quad P_{c}\left(x D_{x} V_{\alpha}^{c}\right)=-c t D_{x} V_{\alpha}^{c}-\left(x+2 b t^{2}\right) D_{x}^{2} V_{\alpha}^{c}$

We can easily obtain this proposition from the definitions of these auxiliary functions. So we omit their proofs. Adding two relations (5) and (6), we have the next proposition.

\section{Proposition 2.}

(1) $\left(t D_{t}+2 x D_{x}\right) U_{\alpha}^{c}=2 \alpha U_{\alpha}^{c}$

(2) $\left(t D_{t}+2 x D_{x}\right) V_{\alpha}^{c}=(2 \alpha+1) V_{\alpha}^{c}$

(3) $D_{x} U_{\alpha}^{c}=U_{\alpha-1}^{c+1}, D_{x} V_{\alpha}^{c}=V_{\alpha-1}^{c+1}$

(1) says that $U_{\alpha}^{c}(t, x)$ is the partially weighted homogeneous function of degree $2 \alpha$, that is, $U_{\alpha}^{c}(t, x)$ satisfies the identity $W\left(\lambda^{2} t, \lambda x\right)=\lambda^{2 \alpha} W(t, x)$. (2) says that $V_{\alpha}^{c}(t, x)$ is the partially weighted homogeneous function of degree $2 \alpha+1$, that is, $V_{x}^{c}(t, x)$ satisfies the identity $W\left(\lambda^{2} t, \lambda x\right)=\lambda^{2 x+1} W(t, x)$. This fact suggests us that we may seek the explicit representations of the auxiliary functions as the form $U_{\alpha}^{c}(t, x)=\frac{\xi^{\alpha}}{\Gamma(\alpha+1)} U(z)$ and $V_{\alpha}^{c}(t, x)=\frac{t \xi^{\alpha}}{\Gamma(\alpha+1)} V(z)$, and then actually we 
reach the hyper-geometric equations of $U(z)$ and respectively $V(z)$. (3) is the most important relations.

For the proof of (1), it is enough to verify that $W(t, x)=\left(t D_{t}+2 x D_{x}\right) U_{\alpha}^{c}(t, x)$ satisfies the Cauchy problem $P_{c} W=0$ with the initial conditions $W(0, x)=$ $2 \alpha f_{\alpha}(x)$ and $D_{t} W(0, x)=0$. For the proof of (2), it is enough to verify that $W(t, x)=\left(t D_{t}+2 x D_{x}\right) V_{\alpha}^{c}(t, x)$ satisfies the Cauchy problems $P_{c} W=0$ with the initial conditions $W(0, x)=0$ and $D_{t} W(0, x)=(2 \alpha+1) f_{\alpha}(x)$. For the proof of (3), it is enough to verify that $D_{x} U_{\alpha}^{c}(t, x)$ and $D_{x} V_{\alpha}^{c}(t, x)$ satisfy respectively the Cauchy problem $\left[D_{x}\left(P_{c}\left(U_{\alpha}^{c}\right)\right)\right]=P_{c+1}\left(D_{x} U_{\alpha}^{c}\right)=0$ with the initial conditions $D_{x} U_{\alpha}^{c}(0, x)=f_{\alpha-1}(x)$ and $D_{t}\left(D_{x} U_{\alpha}^{c}(0, x)\right)=0$, and the Cauchy problem $\left[D_{x}\left(P_{c}\left(V_{\alpha}^{c}\right)\right)\right]=P_{c+1}\left(D_{x} V_{\alpha}^{c}\right)=0$ with the initial conditions $D_{x} V_{\alpha}^{c}(0, x)=0$ and $D_{l}\left(D_{x} V_{\alpha}^{c}(0, x)\right)=f_{\alpha-1}(x)$.

\section{Proposition 3.}

(1) $x U_{\alpha-1}^{c+1}=\alpha U_{\alpha}^{c}-\frac{t}{2} D_{t} U_{\alpha}^{c}$

(2) $x V_{\alpha-1}^{c+1}=\left(\alpha+\frac{1}{2}\right) V_{\alpha}^{c}-\frac{t}{2} D_{t} V_{\alpha}^{c}$

(3) $x D_{t} U_{\alpha-1}^{c+1}=-\frac{b}{2} t^{3} U_{\alpha-2}^{c+2}-\frac{\alpha+c-1}{2} t U_{\alpha-1}^{c+1}+\left(\alpha-\frac{1}{2}\right) D_{t} U_{\alpha}^{c}-\frac{t^{2}}{4} D_{t} U_{\alpha-1}^{c+1}$

(4) $x D_{t} V_{\alpha-1}^{c+1}=-\frac{b}{2} t^{3} V_{\alpha-2}^{c+2}-\frac{1+2(\alpha+c-1)}{4} t V_{\alpha-1}^{c+1}+\alpha D_{t} V_{\alpha}^{c}+\frac{t^{2}}{4} D_{t} U_{\alpha-1}^{c+1}$.

For the proof of (1) and (2), we rewrite (1) and (2) in Proposition 2 using (3) in Proposition 2. By differentiating (1) and (2) with respect $t$ and using these relations, we obtain (3) and (4).

\section{Construction of the solution}

In this section we construct the solution of this Cauchy problem, that is, we determine all holomorphic coefficients $u_{r, k}(t, x), g_{r, k}(t, x), v_{r, k}(t, x)$ and $h_{r, k}(t, x)$.

To verify that the series represents its solution, we substitute the series in $L u=0$ and using the previous propositions we have

$$
\begin{aligned}
L u= & \sum_{r=0}^{\infty} \sum_{k=0}^{r}\left[\left(b t^{2} \Lambda+2 b t\right) g_{r+1, k+1}-(M+k) u_{r+1, k+1}\right. \\
+ & \left.(\alpha+r+c-k-1) \Lambda g_{r, k}+\left(L-2(\alpha+r-1) D_{x}\right) u_{r, k}\right] U_{\alpha+r-1}^{c-k+1} \\
+ & {\left[-\left(\frac{t}{2} \Lambda+\mathrm{M}+k+1\right) g_{r+1, k+1}+\Lambda u_{r+1, k+1}\right.} \\
& \left.+\left(L-2\left(\alpha+r-\frac{1}{2}\right) D_{x}\right) g_{r, k}\right] D_{t} U_{\alpha+r}^{c-k} \\
+ & {\left[\left(b t^{2} \Lambda+2 b t\right) h_{r+1, k+1}-(M+k) v_{r+1, k+1}\right.} \\
& \left.+\left(\alpha+r+c-k-\frac{1}{2}\right) \Lambda h_{r, k}+\left(L-2\left(\alpha+r-\frac{1}{2}\right) D_{x}\right) v_{r, k}\right] V_{\alpha+r-1}^{c-k+1}
\end{aligned}
$$




$$
\begin{gathered}
+\left[-\left(\frac{t}{2} \Lambda+\mathrm{M}+k+1\right) h_{r+1, k+1}+\Lambda v_{r+1, k+1}\right. \\
\left.+\left(L-2(\alpha+r) D_{x}\right) h_{r, k}\right] D_{t} V_{\alpha+r}^{c-k}
\end{gathered}
$$

where $\Lambda=2 D_{t}+t D_{x}-a(t, x)$ and $\mathrm{M}=2 b t^{2} D_{x}+t \tilde{c}(t, x)$. On the other hand, from the initial data in the similar way we have

$$
\begin{aligned}
& u(0, x)=\sum_{r=0}^{\infty} \sum_{k=0}^{r}\left[u_{r, k}(0, x)+h_{r-1, k}(0, x)\right] f_{\alpha+r-1}(x)=w_{1}(x) f_{\alpha}(x) \\
& D_{t} u(0, x)= \sum_{r=0}^{\infty} \sum_{k=0}^{r}\left[v_{r, k}(0, x)+D_{t} u_{r, k}(0, x)\right. \\
&\left.+(\alpha+r+c-k) g_{r, k}(0, x)+D_{t} h_{r-1, k}(0, x)\right] f_{\alpha+r-1}(x) \\
&= w_{2}(x) f_{\alpha}(x) .
\end{aligned}
$$

Setting the coefficients of $U_{\alpha+r-1}^{c-k+1}, D_{t} U_{\alpha+r}^{c-k}, V_{\alpha+r-1}^{c-k+1}, D_{t} V_{\alpha+r}^{c-k}$ and $f_{\alpha+r-1}$ equal to zero, we obtain the recursion formulae for $u_{r, k}, g_{r, k}, v_{r, k}, h_{r, k}$ :

$$
\begin{aligned}
& \Lambda u_{r+1, k+1}-\left(\frac{t}{2} \Lambda+\mathrm{M}+(k+1)\right) g_{r+1, k+1} \\
& \quad=-\left(L-2\left(\alpha+r-\frac{1}{2}\right) D_{x}\right) g_{r, k} \\
& -(\mathrm{M}+k) u_{r+1, k+1}+\left(b t^{2} \Lambda+2 b t\right) g_{r+1, k+1} \\
& \quad=-\left(L-2(\alpha+r-1) D_{x}\right) u_{r, k}-(\alpha+r+c-k-1) \Lambda g_{r, k}
\end{aligned}
$$

(E.2) $\quad \Lambda v_{r+1, k+1}-\left(\frac{t}{2} \Lambda+\mathrm{M}+(k+1)\right) h_{r+1, k+1}$

$$
\begin{aligned}
& \quad=-\left(L-2(\alpha+r) D_{x}\right) h_{r, k} \\
& -(\mathrm{M}+k) v_{r+1, k+1}+\left(b t^{2} \Lambda+2 b t\right) h_{r+1, k+1} \\
& \quad=-\left(L-2\left(\alpha+r-\frac{1}{2}\right) D_{x}\right) v_{r, k}-\left(\alpha+r+c-k-\frac{1}{2}\right) \Lambda h_{r, k}
\end{aligned}
$$

where $\Lambda=2 D_{t}+t D_{x}-a(t, x)$ and $\mathrm{M}=2 b t^{2} D_{x}+t \tilde{c}(t, x)$.

$$
\begin{array}{ll}
\text { (I.D.u) } \quad & \sum_{k=0}^{r}\left[u_{r, k}(0, x)+h_{r-1, k}(0, x)\right]=w_{1}(x) \quad(\text { for } r=1), \\
& \sum_{k=0}^{r}\left[u_{r, k}(0, x)+h_{r-1, k}(0, x)\right]=0 \quad(\text { otherwise }) . \\
\text { (I.D.v) } \quad \sum_{k=0}^{r}\left[v_{r, k}(0, x)+D_{t} u_{r, k}(0, x)\right. & \left.\quad+(\alpha+r+c-k) g_{r, k}(0, x)+D_{t} h_{r-1, k}(0, x)\right]=w_{2}(x) \quad(\text { for } r=1), \\
& \sum_{k=0}^{r}\left[v_{r, k}(0, x)+D_{t} u_{r, k}(0, x)\right. \\
& \left.+(\alpha+r+c-k) g_{r, k}(0, x)+D_{t} h_{r-1, k}(0, x)\right]=0 \quad \text { (otherwise). }
\end{array}
$$


We remark that these first order systems for $u_{r+1, k+1}, g_{r+1, k+1}$ and for $v_{r+1, k+1}$, $h_{r+1, k+1}$ are composed of the first order systems of the same form

$$
\begin{aligned}
\left(b t^{2} \Lambda+2 b t\right) g-(\mathrm{M}+k) u & =F(t, x) \\
-\left(\frac{t}{2} \Lambda+M+(k+1)\right) g+\Lambda u & =G(t, x)
\end{aligned}
$$

where $F(t, x)$ and $G(t, x)$ are holomorphic functions in a neighbourhood of the origin of $C^{2}$ and in particular when $k=0, F(t, x) \equiv 0$.

We note that this first order system $(\mathrm{F})$ and $(\mathrm{G})$ of the case $k=0$ is slightly different from this system of the case $k>0$. By adding $(\mathrm{F})$ to $2 b t \times(\mathrm{G})$, we get

$$
\begin{aligned}
\left(b t^{2} \Lambda+2 b t\right) g-(\mathrm{M}+k) u & =F(t, x) \quad(\mathrm{F}) \\
(2 b t \Lambda-\mathrm{M}-k) u-2 b t(\mathrm{M}+k) g & =F(t, x)+2 b t G(t, x)
\end{aligned}
$$

that is

$$
\begin{gathered}
t\left(2 b t D_{t}+b t^{2} D_{x}-b t a(t, x)+2 b\right) g-\left(t\left(2 b t D_{x}+\tilde{c}(t, x)\right)+k\right) u=F(t, x) \\
\left(t\left(4 b D_{t}-2 b a(t, x)-\tilde{c}(t, x)\right)-k\right) u-t\left(4 b^{2} t^{2} D_{x}+2 b t \tilde{c}(t, x)+2 b k\right) g=F(t, x)+2 b t G(t, x)
\end{gathered}
$$

At first we study this first order recurrence system for $k=0$. In particular when $k=0$, we can rewrite above system in the following form

$$
\begin{gathered}
\left(2 t D_{t}+t^{2} D_{x}-t a(t, x)+2\right) g-\left(2 t D_{x}+\frac{\tilde{c}(t, x)}{b}\right) u=0 \\
\left(4 D_{t}-2 a(t, x)-\frac{\tilde{c}(t, x)}{b}\right) u-\left(4 b t^{2} D_{x}+2 t \tilde{c}(t, x)\right) g=2 G(t, x)
\end{gathered}
$$

with the initial data $u(0, x)$ given.

This is Fuchian system of first order, that we can solve in the routine way, using the method of indetermined coefficients due to Fuchs. There exist unique holomorphic solutions $u(t, x)$ and $g(t, x)$ of this Fuchsian Cauchy problem.

Secondly we study this first order recurrence system for $k>0$. When $k>0$, we note that for the solvability of this first order system of $u(t, x)$ and $g(t, x)$, the following condition is necessary:

$$
-k u(0, x)=F(0, x) .
$$

We shall use the next notation

$$
R f(t, x) \equiv[R f](t, x) \equiv \frac{1}{t}(f(t, x)-f(0, x)) .
$$

Now we apply this necessary condition to (E.1) and (E.2) and then we can rewrite this necessary condition in the following form: for $k>0$ 
(I.D.1) $-k u_{r+1, k+1}(0, x)$

$$
=\left(-\left.\left(L-2(\alpha+r-1) D_{x} u_{r, k}-(\alpha+r+c-k-1) \Lambda g_{r, k}\right)\right|_{t=0}\right.
$$

(I.D.2) $-k v_{r+1, k+1}(0, x)$

$$
=\left(-\left.\left(L-2\left(\alpha+r-\frac{1}{2}\right) D_{x} v_{r, k}-\left(\alpha+r+c-k-\frac{1}{2}\right) \Lambda h_{r, k}\right)\right|_{t=0} .\right.
$$

So $u_{r+1, k+1}(0, x)$ and $v_{r+1, k+1}(0, x)$ are determined by $u_{r, k}, g_{r, k}$ and $v_{r, k}, h_{r, k}$ respectively. then (E.1) and (E.2) become

$$
\begin{aligned}
& (t \Lambda+2) g-\left(\frac{1}{b t} \mathrm{M}+\frac{k}{b} R\right) u=\frac{1}{b} R F(t, x) \\
& \Lambda u-\left(\frac{t}{2} \Lambda+\mathrm{M}+(k+1)\right) g=G(t, x)
\end{aligned}
$$

where $F(t, x)$ and $G(t, x)$ are holomorphic functions in a neighbourhood of the origin of $C^{2}$ and $\Lambda=2 D_{t}+t D_{x}-a(t, x)$ and $\mathrm{M}=t\left(2 b t D_{x}+\tilde{c}\right)$.

We can solve this first order system under the Condition (b), that is, Poincare condition for this system. In fact we set $u=\sum_{j=0}^{\infty} u_{j}(x) t^{j}$ and $g=\sum_{j=0}^{\infty} g_{j}(x) t^{j}$ and other functions into the similar Taylor expansion form, and then we substitute these series into the corresponding place in this system respectively. After the calculation setting the coefficients of $t^{j}$ equal to zero, we obtain recursion formulae to determinate the coefficients $u_{j}(x)$ and $g_{j}(x)$ inductively. These recursion formulae are first order linear system for $u_{j}(x)$ and $g_{j}(x)$ whose determinant is $b-b(j, k)$ where $b(j, k)=\frac{k(k+1+j)}{4(j+1)^{2}}$. If $b \neq b(j, k)$, we can determine all coefficients $u_{j}(x)$ and $g_{j}(x)$ inductively. This condition $b \neq b(j, k)$, is equal to $1+16 b \neq 1+16 b(j, k)=\frac{(2 k+j+1)^{2}}{(j+1)^{2}}$. By the definition $D=\sqrt{16 b+1}$, this is equal to the condition $D \neq 1+\frac{2 k}{j+1}$ for all non-negative integers $j, k \cdot \frac{2 k}{j+1}$ can be any non-negative rational number and so Poincare condition $b \neq b(j, k)$ is equal Condition (b). Namely under Condition (b) there exist unique holomorphic solutions $u(t, x)$ and $g(t, x)$ in the neighbourhood of the origin of $C^{2}$.

By these facts holomorphic coefficients $u_{r, k}(t, x), g_{r, k}(t, x), v_{r, k}(t, x)$ and $h_{r, k}(t, x)$ can be determined inductively.

In fact first step: $u_{0,0}(0, x)$ is determined from the relation of (I.D.u) and initial data $w_{1}(x)$ and with this $u_{0,0}(0, x)$ as the initial data we can solve the Cauchy problem of the first order Fuchsian system (E.1) for $u_{0,0}(t, x)$ and $g_{0,0}(t, x)$. and then we can determine $v_{0,0}(0, x)$ from the relation of (I.D.v) and initial data $w_{2}(x), u_{0.0}(t, x), g_{0,0}(t, x)$ and using this $v_{0,0}(0, x)$ as the initial data we can solve the Cauchy problem of the first order Fuchsian system (E.2) for $v_{0,0}(t, x)$ and $h_{0,0}(t, x)$. Thus we can determine the holomorphic coefficients $\left\{u_{0,0}(t, x), g_{0,0}(t, x), v_{0,0}(t, x), h_{0,0}(t, x)\right\}$. 
Second step: first we suppose all holomorphic coefficients $u_{r, k}, g_{r, k}, v_{r, k}$ and $h_{r, k}(0 \leq r \leq R, 0 \leq k \leq K)$ are determined, and then we can solve the Cauchy problems of the first order Fuchsian systems (E.1) and (E.2) for $u_{R+1, k}(t, x)$, $g_{R+1, k}(t, x)(1 \leq k \leq K+1)$ and $v_{R+1, k}(t, x), h_{R+1, k}(t, x)(1 \leq k \leq K+1)$ with the corresponding initial data (I.D.1) and (I.D.2) respectively, and so we can restrict these holomorphic coefficients on the initial surface $\{t=0\}$ and then we can determine $u_{R+1,0}(0, x)$ from the relation (I.D.u) and using this $u_{R+1,0}(0 . x)$ as the initial data we can solve the first order Fuchsian Cauchy problem of (E.1) for $u_{R+1,0}(t, x)$ and $g_{R+1,0}(t, x)$. We can restrict $u_{R+1,0}(t, x)$ and $g_{R+1,0}(t, x)$ on the initial surface $\{t=0\}$, and using this $u_{R+1.0}(0, x)$ and $g_{R+1,0}(0, x)$ we can determine $v_{R+1,0}(0, x)$, which we use as the initial data for the first order Fuchsian Cauchy problem of (E.1) for $v_{R+1,0}(t, x)$ and we can solve it. Finally due to mathematical induction we can determine all holomorphic coefficients $u_{r, k}(t, x), g_{r, k}(t, x), v_{r, k}(t, x)$ and $h_{r, k}(t, x)(0 \leq r \leq R+1,0 \leq k \leq K+1)$. We shall prove these holomorphic coefficients $u_{r, k}(t, x), g_{r, k}(t, x), v_{r, k}(t, x)$ and $h_{r, k}(t, x)$ have a common existence domain and suitable estimates in that domain, which lead the convergence of the expansion of the series of the formal solution in the next section.

\section{Convergence of the formal series}

For the method of the majoration, we introduce families of the scale functions $\phi_{\alpha}(t, x)$ as follows

$$
\begin{aligned}
\phi(t, x)=(\sqrt{2(R-x)}-\rho t)^{-1}=\sum_{j=0}^{\infty} \frac{(\rho t)^{j}}{(\sqrt{2(R-x)})^{j+1}} . \\
\phi_{\alpha}(t, x)=D_{x}^{\alpha} \phi(t, x) \\
=\sum_{j=0}^{\infty}(j+1)(j+3) \cdots(j+2 \alpha-1)(2(R-x))^{(j+2 x+1) /(-2)}(\rho t)^{j} \\
\quad \text { for }|x|\langle R, \rho\rangle 1,|\rho t|^{2}\langle|2(R-x)|
\end{aligned}
$$

We put $R[f(t, x)]=t^{-1}(f(t, x)-f(0, x)$. The following propositions can be easily verified. See [U.1].

Proposition $\phi . \quad\left\{\phi_{\alpha}(t, x)\right\}$ have following properties.

(1) $D_{t} \phi_{\alpha} \gg \rho t \phi_{\alpha+1}$

(2) $D_{t}^{2} \phi_{\alpha} \gg \rho^{2} \phi_{\alpha+1}$

(3) $D_{t}^{2} \phi_{\alpha} \gg \rho^{2} t^{2} \phi_{\alpha+2}$

(4) $\left(t D_{t}+1\right) \phi_{\alpha+1} \gg D_{t} \phi_{x}, R\left[D_{t} \phi_{x}\right], D_{t}^{2} \phi_{x}$

(5) $8 \rho\left(t D_{t}+1\right) \phi_{\alpha+2} \gg D_{t}^{3} \phi_{x}, R\left[D_{t}^{3} \phi_{x}\right]$

(6) $2 \phi_{\alpha+1}(0, x) \gg D_{t}^{2} \phi_{\alpha}(0, x)$

(7) $4 D_{t}^{2} \phi_{\alpha+1}(0, x) \gg D_{t}^{4} \phi_{\alpha}(0, x)$

(8) $\frac{1}{\left(R^{\prime}-x\right)\left(R^{\prime \prime}-t\right)} D_{t}^{k} \phi_{\alpha} \ll \frac{1}{\left(R^{\prime}-R\right)\left(R^{\prime \prime}-R\right)} D_{t}^{k} \phi_{\alpha} \quad \alpha \geq 1, R \leq 1, R^{\prime \prime}, R^{\prime}>R$ 
The majoration method applied to the proof of the convergence of the formal solution is based on the next proposition

Proposition (M.R.) Under Condition (b) for the Cauchy problem

$$
\begin{aligned}
& (t \Lambda+2) g-\left(\frac{1}{b t} \mathrm{M}+\frac{k}{b} R\right) u=\frac{1}{b} R F(t, x) \\
& \Lambda u-\left(\frac{t}{2} \Lambda+\mathrm{M}+(k+1)\right) g=G(t, x) \quad \text { with the initial data } u(0, x)=u_{0}(x),
\end{aligned}
$$

where $\Lambda=2 D_{t}+t D_{x}-a(t, x), \mathbf{M}=2 b t^{2} D_{x}+t \tilde{c}(t, x)$ and $a(t, x), \tilde{c}(t, x), F(t, x)$ and $G(t, x)$ are holomorphic functions in a neighbourhood of the origin of $C^{2}$, there exist unique holomorphic solutions $u(t, x)$ and $g(t, x)$ in the neighbourhood of the origin. Moreover assuming $a(t, x) \ll \tilde{a}(t, x), \quad \tilde{c}(t, x) \ll \tilde{\tilde{c}}(t, x), \quad F(t, x) \ll \tilde{F}(t, x), \quad G(t, x) \ll$ $\tilde{G}(t, x)$ and $u_{0}(x) \ll \tilde{u}_{0}(x)$, we can verify that $u(t, x) \ll \tilde{u}(t, x)$ and $g(t, x) \ll \tilde{g}(t, x)$ if $\tilde{u}(t, x)$ and $\tilde{g}(t, x)$ satisfy

$$
\begin{aligned}
\left(2 t D_{t}+2\right) \tilde{g}(t, x) & \gg\left(t D_{x}+\tilde{a}(t, x)\right) \tilde{g}(t, x)+\left(2 t D_{x}+\frac{\tilde{\tilde{c}}(t, x)}{|b|}+\frac{k}{|b|}\right) \tilde{u}(t, x)+\frac{1}{|b|} R F(t, x) \\
2 D_{t} \tilde{u}(t, x) & \gg\left(t D_{x}+\tilde{a}(t, x)\right) \tilde{u}(t, x)+\left(\frac{t}{2} \tilde{\Lambda}+\tilde{M}+(k+1)\right) \tilde{g}(t, x)+\tilde{G}(t, x) \\
\tilde{u}(0, x) & \gg \tilde{u}_{0}(x)
\end{aligned}
$$

where $\tilde{\Lambda}=2 D_{t}+t D_{x}+\tilde{a}(t, x)$ and $\mathrm{M}=2|b| t^{2} D_{x}+\tilde{\tilde{c}}(t, x)$.

Applying these propositions to the recurrence formulae (E.1) and (E.2) with (I.D.u) and (I.D.v), we have the following proposition says that these holomorphic coefficients $u_{r, k}(t, x), g_{r, k}(t, x), v_{r, k}(t, x)$ and $h_{r, k}(t, x)$ have a common existence domain and suitable estimates in that domain.

Proposition (Es.) There exist positive constants $U, G, V, H, K, L, R$ and $\rho$ independent of $\alpha, r, k$ such that

$$
\begin{aligned}
u_{r, k}(t, x) & \ll U \frac{1}{(k-1) !(r-k+1) !} \frac{K^{\alpha+r}}{L^{k}} D_{t} \phi_{\alpha+r+k+1}(t, x) \\
g_{r, k}(t, x) & \ll G \frac{1}{k !(r-k) !} \frac{K^{\alpha+r+1}}{L^{k}} \phi_{\alpha+r+k+2}(t, x) \\
v_{r, k}(t, x) & \ll V \frac{1}{(k-1) !(r-k+1) !} \frac{K^{\alpha+r}}{L^{k}} D_{t} \phi_{\alpha+r+k+2}(t, x) \\
h_{r, k}(t, x) & \ll H \frac{1}{k !(r-k) !} \frac{K^{\alpha+r+1}}{L^{k}} \phi_{\alpha+r+k+3}(t, x) .
\end{aligned}
$$

From this proposition, we know holomorphic coefficients $u_{r, k}(t, x), g_{r, k}(t, x)$, $v_{r, k}(t, x)$ and $h_{r, k}(t, x)$ have a common existence domain that is a neighbourhood of 
the origin and the following estimates in that domain of the base space

$$
\begin{aligned}
\left|u_{r, k}\right| & \leq C \frac{1}{(k-1) !(r-k+1) !} \frac{K^{r}}{L^{k}} \Gamma(r+k+1) \\
\left|g_{r, k}\right| & \leq C \frac{1}{k !(r-k) !} \frac{K^{r}}{L^{k}} \Gamma(r+k+1) \\
\left|v_{r, k}\right| & \leq C \frac{1}{(k-1) !(r-k+1) !} \frac{K^{r}}{L^{k}} \Gamma(r+k+2) \\
\left|h_{r, k}\right| & \leq C \frac{1}{k !(r-k) !} \frac{K^{r}}{L^{k}} \Gamma(r+k+2)
\end{aligned}
$$

where $C, K, L$ are positive constants independent of $r, k$.

On the other hand in Appendix we have the estimates on any compact set $\tilde{K}$ in the universal covering space $\tilde{\omega}_{\lambda}$ over $\omega_{\lambda}=\{(t, x): 0 \neq|\xi|<\lambda, 0 \neq|\eta|<\lambda\}$

$$
\begin{gathered}
\left|U_{\alpha+r-1}^{c-k+1}(l(P))\right|,\left|\tilde{U}_{\alpha+r-1}^{c-k+1}(l(P))\right|,\left|V_{\alpha+r-1}^{c-k+1}(l(P))\right|, \\
\left|\tilde{V}_{\alpha+r-1}^{c-k+1}(l(P))\right| \leq C(\alpha, c, \tilde{K}) T_{1}^{r} T_{2}^{k} \frac{(r-k) !}{r !} \lambda^{r}
\end{gathered}
$$

where $T_{1}, T_{2}, C(\alpha, c, \tilde{K})$ are positive constants and the common factor independent of $r, k$.

According these estimates, choosing $\lambda>0$ sufficiently small, we can prove the convergence df the formal solution on the universal covering space $\tilde{\omega}_{\lambda}$ over $\omega_{\lambda}=\{(t, x): 0 \neq|\xi|<\lambda, 0 \neq|\eta|<\lambda\}$ which we can employ as $\omega$-K in our theorem. We note that uniqueness of the solution is due to the CauchyKobalevskaya theorem. Thus we can prove our theorem.

\section{Appendix}

In this appendix we study the ramification, the singularities and the estimates of the auxiliary functions $U_{\alpha}^{c}(t, x)$ and $V_{\alpha}^{c}(t, x)$. We treat these auxiliary functions in the more general form but in this paper we use only the results of the case $n=1$.

To be precise, we put

$$
P_{c}=D_{t}^{2}-\left(x t^{n-1}+b t^{2 n}\right) D_{x}^{2}-c t^{n-1} D_{x}
$$

where $b$ and $c$ are complex parameters and $n$ is a natural number.

We first introduce $U_{\alpha}^{c}(t, x)$ and $V_{\alpha}^{c}(t, x)$ as the solutions of the following Cauchy problems respectively:

$$
\begin{gathered}
P_{c}\left[U_{\alpha}^{c}(t, x)\right]=\left(D_{t}^{2}-\left(x t^{n-1}+b t^{2 n}\right) D_{x}^{2}-c t^{n-1} D_{x}\right) U_{\alpha}^{c}(t, x) \\
\text { with the initial data } U_{\alpha}^{c}(0, x)=f_{\alpha}(x) \\
D_{t} U_{\alpha}^{c}(0, x)=0,
\end{gathered}
$$




$$
\begin{gathered}
P_{c}\left[V_{\alpha}^{c}(t, x)\right]=\left(D_{t}^{2}-\left(x t^{n-1}+b t^{2 n}\right) D_{x}^{2}-c t^{n-1} D_{x}\right) V_{\alpha}^{c}(t, x) \\
\text { with the initial data } V_{\alpha}^{c}(0, x)=f_{\alpha}(x) \\
D_{t} V_{\alpha}^{c}(0, x)=f_{\alpha}(x)
\end{gathered}
$$

And we introduce $\xi(t, x)$ and $\eta(t, x)$ as two solutions of the following Cauchy problem for the characteristic equation of the operator $P_{c}$ respectively.

$$
\varphi_{t}^{2}-\left(x t^{n-1}+b t^{2 n}\right) \varphi_{x}^{2}=-t^{n-1} \varphi \quad \text { with the initial data } \varphi(0, x)=x .
$$

We can solve this problem explicitly:

$$
\begin{aligned}
& \xi(t, x)=x-\frac{1}{2(n+1)^{2}}(1+D) t^{n+1} \\
& \eta(t, x)=x-\frac{1}{2(n+1)^{2}}(1-D) t^{n+1}
\end{aligned}
$$

where $D=\sqrt{1+4(n+1)^{2} b} \operatorname{Re}(D) \geq 0$.

To seek $U_{\alpha}^{c}(t, x)$, we put $U_{\alpha}^{c}(t, x)=\frac{\xi^{\alpha}}{\Gamma(\alpha+1)} f(z)$ where $z=1-\frac{\eta}{\xi}$ and we substitute the right hand side in $P_{c}\left[U_{\alpha}^{c}\right]=0$. Then we have

$$
\begin{aligned}
& z(1-z) f^{\prime \prime}+\left[\frac{n}{n+1}-\left(1-\alpha+\frac{n}{2(n+1)}\left(1+\frac{1}{D}\right)+\frac{c+\alpha-1}{D}\right) z\right] f^{\prime} \\
& -(-\alpha)\left(\frac{n}{2(n+1)}\left(1+\frac{1}{D}\right)+\frac{c+\alpha-1}{D}\right) f=0 \quad \text { with the initial data } f(0)=1 .
\end{aligned}
$$

This is the hypergeometric equation and so we have

$$
f(z)=F\left(-\alpha, \frac{n}{2(n+1)}\left(1+\frac{1}{D}\right)+\frac{c+\alpha-1}{D}, \frac{n}{n+1} ; z\right) .
$$

In the similar way we can seek $V_{\alpha}^{c}(t, x)$. We put $V_{\alpha}^{c}(t, x)=\frac{t \xi^{\alpha}}{\Gamma(\alpha+1)} f(z)$ and substitute the right hand side in $P_{c}\left[V_{\alpha}^{c}\right]=0$. Then we have

$$
\begin{aligned}
& z(1-z) f^{\prime \prime}+\left[\frac{n+2}{n+1}-\left(1-\alpha+\frac{n+2}{2(n+1)}\left(1+\frac{1}{D}\right)+\frac{c+\alpha-1}{D}\right) z\right] f^{\prime} \\
& -(-\alpha)\left(\frac{n+2}{2(n+1)}\left(1+\frac{1}{D}\right)+\frac{c+\alpha-1}{D}\right) f=0 \quad \text { with the initial data } f(0)=1 .
\end{aligned}
$$

So we have

$$
f(z)=F\left(-\alpha, \frac{n+2}{2(n+1)}\left(1+\frac{1}{D}\right)+\frac{c+\alpha-1}{D}, \frac{n+2}{n+1} ; z\right) .
$$


We know explicit representations of $U_{\alpha}^{c}(t, x)$ and $V_{\alpha}^{c}(t, x)$ as follows

$$
\begin{aligned}
& U_{\alpha}^{c}(t, x)=\frac{\xi^{\alpha}}{\Gamma(\alpha+1)} F\left(-\alpha, \frac{n}{2(n+1)}\left(1+\frac{1}{D}\right)+\frac{c+\alpha-1}{D}, \frac{n}{n+1} ; z\right) \\
& V_{\alpha}^{c}(t, x)=\frac{t \xi^{\alpha}}{\Gamma(\alpha+1)} F\left(-\alpha, \frac{n+2}{2(n+1)}\left(1+\frac{1}{D}\right)+\frac{c+\alpha-1}{D}, \frac{n+2}{n+1} ; z\right) .
\end{aligned}
$$

Because $U_{x}^{c}(t, x)$ and $V_{x}^{c}(t, x)$ have hypergeometric functions as important factors respectively, the study of the ramification and singularities and multivaluedness of these functions are reduced to those of hypergeometric functions. The next lemma about the analytic continuation that is the monodromy theory of the hypergeometric functions, plays a fundamental role in the study of the behaviour of these functions $U_{x}^{c}(t, x)$ and $V_{\alpha}^{c}(t, x)$ around the branching surfaces.

Lemma A.1 (see [Ki.1]). Let $S$ be the Riemann sphere. Put $D=S-$ $\{0,1, \infty\}$. Set $F=F(A, B, C ; z)$ and $\tilde{F}=z^{1-c} F(A-C+1, B-C+1,2-C ; z)$ which constitute a fundamental system of solutions for the hyper-geometric ordinary differential equation $\left[z(1-z) \frac{d^{2}}{d z^{2}}+\{C-(A+B+1) z\} \frac{d}{d z}-A B\right] u(z)=0 . \quad$ Now the monodromy representation $\rho$ of the hyper-geometric ordinary differential equation with respect to the fundamental system $\{F, \tilde{F}\}$ is defined in the following way:

Denote by $l_{0}\left(l_{1}, l_{\infty}\right.$ respectively) a loop which encircles the point $0(1, \infty$ respectively) once in the positive sense. We denote by the same letter $l_{0}\left(l_{1}, l_{\infty}\right.$ respectively) a homotopy class containing $l_{0}\left(l_{1}, l_{\infty}\right.$ respectively). Let $\pi=\pi(D)$ be the fundamental group of $D$. Then we can define a homomorphism $\rho$ of the group $\pi$ onto the group $G \subset G L(2, C)$ which is called the monodromy representation of the hyper-geometric differential equation with respect to the fundamental system $\{F, \tilde{F}\}$, where $G$ is the subgroup of $G L(2, C)$, generated by $g_{0}$ and $g_{1}$,

$$
\begin{gathered}
\rho\left(l_{0}\right)=g_{0}=\left(\begin{array}{cc}
1 & 0 \\
0 & e^{-2 \pi i C}
\end{array}\right) \\
\rho\left(l_{\propto}\right)=g_{\propto}=\left(g_{0} g_{1}\right)^{-1} \\
\rho\left(l_{1}\right)=g_{1}=\hat{D}^{-1} \hat{C}^{-1}\left(\begin{array}{cc}
1 & 0 \\
0 & e^{2 \pi i(C-B-A)}
\end{array}\right) \hat{C} \hat{D} \\
\text { where } \hat{C}=\left(\begin{array}{cc}
e^{-2 \pi i A}-e^{-2 \pi i C} & e^{-2 \pi i(C-B)}\left(e^{-2 \pi i A}-1\right) \\
e^{-2 \pi i B}-1 & 1-e^{-2 \pi i(C-B)}
\end{array}\right) \\
\hat{D}=\left(\begin{array}{cc}
\frac{\Gamma(B) \Gamma(C-B)}{\Gamma(C)} & 0 \\
0 & \frac{\Gamma(A-C+1) \Gamma(1-A)}{\Gamma(2-C)} e^{\pi i(C+A-B-1)}
\end{array}\right)
\end{gathered}
$$




$$
\begin{aligned}
& \hat{C}^{-1}=\frac{1}{|\hat{C}|}\left(\begin{array}{cc}
1-e^{-2 \pi i(C-B)} & 1-e^{-2 \pi i B} \\
e^{-2 \pi i(C-B)}\left(1-e^{-2 \pi i A}\right) & e^{-2 \pi i A}-e^{-2 \pi i C}
\end{array}\right) \\
& |\hat{C}|=e^{-2 \pi i A}\left(1-e^{-2 \pi i C}\right)\left(1-e^{-2 \pi i(C-B-A)}\right) .
\end{aligned}
$$

Namely if $\{F, \tilde{F}\}$ is continued analytically along $l_{0}\left(l_{1}, l_{\infty}\right.$ respectively), then $\left(\begin{array}{c}F \\ \tilde{F}\end{array}\right)$ goes to $g_{0}\left(\begin{array}{c}F \\ \tilde{F}\end{array}\right)\left(g_{1}\left(\begin{array}{c}F \\ \tilde{F}\end{array}\right), g_{\infty}\left(\begin{array}{c}F \\ \tilde{F}\end{array}\right)\right.$ respectively $)$.

We apply this lemma to the study of $U_{\alpha}^{c}(t, x)$ and $V_{\alpha}^{c}(t, x)$.

In the case of $U_{\alpha}^{c}(t, x), \quad F$ is $F(\alpha ; \xi, \eta)=F\left(-\alpha, \frac{n}{2(n+1)}\left(1+\frac{1}{D}\right)+\right.$ $\left.\frac{c+\alpha-1}{D}, \frac{n}{n+1} ; z\right) \quad$ and $\quad$ so $\quad \tilde{F} \quad$ is $\quad \tilde{F}(\alpha ; \xi, \eta)=z^{1 /(n+1)} F\left(\frac{1}{n+1}-\alpha, \frac{1}{n+1}+\right.$ $\left.\frac{n}{2(n+1)}\left(1+\frac{1}{D}\right)+\frac{c+\alpha-1}{D}, \frac{n+2}{n+1} ; z\right)$.

In the case of $V_{\alpha}^{c}(t, x), \quad F \quad$ is $F(\alpha ; \xi, \eta)=F\left(-\alpha, \frac{n+2}{2(n+1)}\left(1+\frac{1}{D}\right)+\right.$ $\left.\frac{c+\alpha-1}{D}, \frac{n+2}{n+1} ; z\right) \quad$ and $\quad$ so $\quad \tilde{F} \quad$ is $\quad \tilde{F}(\alpha ; \xi, \eta)=z^{-1 /(n+1)} F\left(\frac{-1}{n+1}-\alpha, \frac{-1}{n+1}+\right.$ $\left.\frac{n+2}{2(n+1)}\left(1+\frac{1}{D}\right)+\frac{c+\alpha-1}{D}, \frac{n}{n+1} ; z\right)$. To explain the ramification, the multivaluedness and the singularities of $U_{\alpha}^{c}(t, x)$ and $V_{\alpha}^{c}(t, x)$ in terms of the monodromy theory of the hyper-geometric functions, we introduce the new functions $\tilde{U}_{\alpha}^{c}(t, x)=\frac{\xi^{\alpha}}{\Gamma(\alpha+1)} \tilde{F}$ and $\tilde{V}_{\alpha}^{c}(t, x)$ :

$$
\begin{aligned}
& \tilde{U}_{\alpha}^{c}(t, x)=\frac{\xi^{\alpha}}{\Gamma(\alpha+1)} z^{1 /(n+1)} F\left(\frac{1}{n+1}-\alpha, \frac{1}{n+1}+\frac{n}{2(n+1)}\left(1+\frac{1}{D}\right)+\frac{c+\alpha-1}{D}, \frac{n+2}{n+1} ; z\right) \\
& \tilde{V}_{\alpha}^{c}(t, x)=\frac{t \xi^{\alpha}}{\Gamma(\alpha+1)} z^{-1 /(n+1)} F\left(\frac{-1}{n+1}-\alpha, \frac{-1}{n+1}+\frac{n+2}{2(n+1)}\left(1+\frac{1}{D}\right)+\frac{c+\alpha-1}{D}, \frac{n}{n+1} ; z\right) .
\end{aligned}
$$

Let $\omega_{r}=\{(\xi, \eta) ; \xi \neq 0, \eta \neq 0, \xi \neq \eta,|\xi|<r,|\eta|<r\}$. Let $P$ be any point belonging to $\omega_{r}$ and keep $P$ fixed. We consider any loop $l$ starting and terminating at $P$ in the domain $\omega_{r}$. In particular we denote $l(P)$ the terminating point in order to distinguish the terminating point from the starting point $P$. We denote by the same letter $l$ the homotopy class containing $l$ too. Let $\pi=\pi\left(\omega_{r}, P\right)$ be the fundamental group of $\omega_{r}$ with the base point $P$. Denote by $l_{\infty} \in \pi\left(l_{1} \in \pi\right.$, $l_{0} \in \pi$ respectively) a loop which encircles $\xi=0(\eta=0, \xi=\eta$ respectively) once in the positive sense, where by "positive sense" we mean that the loop which is transformed by the mapping $z=1-\frac{\eta}{\xi}$ encircles the corresponding point in the positive sense in $z$-plane. We note that this mapping $z=1-\frac{\eta}{\xi}$ transforms $\xi=0$, 
$\eta=0, \xi=\eta$ to $z=\infty, 1,0$ respectively and $l_{0}, l_{1}, l_{\infty}$ to the corresponding loops in the lemma A.1. Using lemma A.1 we have

$$
\begin{aligned}
\left(\begin{array}{c}
F\left(\alpha ; l_{0}(P)\right) \\
\tilde{F}\left(\alpha ; l_{0}(P)\right)
\end{array}\right) & =g_{0}\left(\begin{array}{c}
F(\alpha ; P) \\
\tilde{F}(\alpha ; P)
\end{array}\right), \quad\left(\begin{array}{c}
F\left(\alpha ; l_{1}(P)\right) \\
\tilde{F}\left(\alpha ; l_{1}(P)\right)
\end{array}\right)=g_{1}\left(\begin{array}{c}
F(\alpha ; P) \\
\tilde{F}(\alpha ; P)
\end{array}\right) \\
\left(\begin{array}{c}
F\left(\alpha ; l_{\infty}(P)\right) \\
\tilde{F}\left(\alpha ; l_{\infty}(P)\right)
\end{array}\right) & =g_{\propto}\left(\begin{array}{c}
F(\alpha ; P) \\
\tilde{F}(\alpha ; P)
\end{array}\right) .
\end{aligned}
$$

Generally we have $\left(\begin{array}{c}F(\alpha ; l(P)) \\ \tilde{F}(\alpha ; l(P))\end{array}\right)=\rho(l)\left(\begin{array}{c}F(\alpha ; P) \\ \tilde{F}(\alpha ; P)\end{array}\right)$.

Therefore we have $\left(\begin{array}{c}U_{\alpha}^{c}\left(l_{0}(P)\right) \\ \tilde{U}_{\alpha}^{c}\left(l_{0}(P)\right)\end{array}\right)=g_{0}\left(\begin{array}{c}U_{\alpha}^{c}(P) \\ \tilde{U}_{\alpha}^{c}(P)\end{array}\right)$

$$
\begin{aligned}
& \left(\begin{array}{c}
U_{\alpha}^{c}\left(l_{1}(P)\right) \\
\tilde{U}_{\alpha}^{c}\left(l_{1}(P)\right)
\end{array}\right)=g_{1}\left(\begin{array}{c}
U_{\alpha}^{c}(P) \\
\tilde{U}_{\alpha}^{c}(P)
\end{array}\right) \\
& \left(\begin{array}{c}
U_{\alpha}^{c}\left(l_{\infty}(P)\right) \\
\tilde{U}_{\alpha}^{c}\left(l_{\infty}(P)\right)
\end{array}\right)=e^{2 \pi i \alpha} g_{\infty}\left(\begin{array}{c}
U_{\alpha}^{c}(P) \\
\tilde{U}_{\alpha}^{c}(P)
\end{array}\right)
\end{aligned}
$$

We introduce the monodromy representation $\rho^{*}$ with respect the pair $\left\{U_{\alpha}^{c}, \tilde{U}_{\alpha}^{c}\right\}$ as a homomorphism $\rho^{*}$ of the group $\pi$ onto the group $G^{*} \subset G L(2, C)$, where $G^{*}$ is the sub-group of $G L(2, C)$ generated by $g_{0}, g_{1}$ and $g_{\infty}^{*}=e^{2 \pi i x} g_{\infty}$ :

$$
\rho^{*}\left(l_{0}\right)=g_{0}, \quad \rho^{*}\left(l_{1}\right)=g_{1}, \quad \rho^{*}\left(l_{\infty}\right)=g_{\infty}^{*} .
$$

Then generally we have

$$
\left(\begin{array}{c}
U_{\alpha}^{c}(l(P)) \\
\tilde{U}_{\alpha}^{c}(l(P))
\end{array}\right)=\rho^{*}(l)\left(\begin{array}{c}
U_{\alpha}^{c}(P) \\
\tilde{U}_{\alpha}^{c}(P)
\end{array}\right)
$$

By the way we need only the behaviour of $U_{\alpha}^{c}(l(P))$. For the study of $U_{\alpha}^{c}(l(P))$, we have to study $\rho^{*}(l)$ namely $g_{0}, g_{1}, g_{\infty}^{*}, \hat{C}$ and $\hat{D}$. Seeing that both $g_{0}$ and $\hat{D}$ are diagonal matrices, they are commutative each other and so $g_{0}=$ $\hat{D}^{-1} g_{0} \hat{D}$. On the other hand $g_{1}$ and $g_{\infty}$ are also in the form $\hat{D}^{-1} \hat{G} \hat{D}$ that is $g_{1}=\hat{D}^{-1} \hat{E}_{1} \hat{D}$ and $g_{\propto}^{*}=\hat{D}^{-1} \hat{E}_{\propto} \hat{D}$. Therefore $\rho^{*}(l)=\hat{D}^{-1} \rho^{\#}(l) \hat{D}$ holds where $\rho^{\#}(l)$ is a homomorphism $\pi$ onto $G^{\#} \subset G L(2, C)$, the subgroup of $G L(2, C)$ generated by $g_{0}, \hat{E}_{1}$ and $\hat{E}_{\infty} . \rho^{\#}(l)$, the principal part of $\rho^{*}(l)$, is to be investigated precisely. $\hat{E}_{\propto}=\hat{E}_{1}^{-1} g_{0}^{-1}$ and $g_{0}$ is a simple diagonal matrix and so the study of $\rho^{\#}(l)$ is reduced to the study of $\hat{E}_{1}$ and $\hat{E}_{1}^{-1}$.

Put $\hat{C}=\left(\begin{array}{ll}\hat{C}_{11} & \hat{C}_{12} \\ \hat{C}_{21} & \hat{C}_{22}\end{array}\right)$ and then we have $\hat{C}^{-1}=\frac{1}{|\hat{C}|}\left(\begin{array}{cc}\hat{C}_{22} & -\hat{C}_{12} \\ -\hat{C}_{21} & \hat{C}_{11}\end{array}\right)$. Put $\hat{G}=\hat{C}^{-1}\left(\begin{array}{ll}1 & 0 \\ 0 & \kappa\end{array}\right) \hat{C}$ where $\hat{G}=\hat{E}_{1}$ or $\hat{E}_{1}^{-1}$, and then 


$$
\begin{aligned}
\hat{G} & =\frac{1}{|\hat{C}|}\left(\begin{array}{cc}
\hat{C}_{11} \hat{C}_{22}-\kappa \hat{C}_{12} \hat{C}_{21} & (1-\kappa) \hat{C}_{12} \hat{C}_{22} \\
(\kappa-1) \hat{C}_{11} \hat{C}_{21} & \kappa \hat{C}_{11} \hat{C}_{22}-\hat{C}_{12} \hat{C}_{21}
\end{array}\right) \\
& =\hat{G}=\frac{1}{|\hat{C}|}\left(\begin{array}{cc}
|\hat{C}|+(1-\kappa) \hat{C}_{12} \hat{C}_{21} & (1-\kappa) \hat{C}_{12} \hat{C}_{22} \\
-(1-\kappa) \hat{C}_{11} \hat{C}_{21} & |\hat{C}|-(1-\kappa) \hat{C}_{11} \hat{C}_{22}
\end{array}\right)
\end{aligned}
$$

where $\kappa=\left\{\begin{array}{l}e^{2 \pi i(C-B-A)} \cdots \cdots \hat{G}=\hat{E}_{1} \\ e^{-2 \pi i(C-B-A)} \cdots \cdot \hat{G}=\hat{E}_{1}^{-1}\end{array}\right.$

So $\hat{G}$ can have the singularities on $|\hat{C}|=e^{-2 \pi i A}\left(1-e^{-2 \pi i C}\right)\left(1-e^{-2 \pi i(C-B-A)}\right)=$ 0 . In the case of $U_{\alpha}^{c}, C=\frac{n}{n+1}$ and in the case of $V_{\alpha}^{c}, C=\frac{n+2}{n+1}$ so that $1-$ $e^{2 \pi i C} \neq 0$. Therefore we can find the singularities on $1-e^{-2 \pi i(C-B-A)}=0$.

$$
1-e^{-2 \pi i(C-B-A)}=\left\{\begin{array}{l}
\frac{-1}{\kappa}(1-\kappa) \cdots \cdots \hat{G}=\hat{E}_{1} \\
(1-\kappa) \cdots \cdots \cdots \cdot \hat{G}=\hat{E}_{1}^{-1}
\end{array}\right.
$$

Put $\hat{G}=\frac{1}{|\hat{C}|}\left(\begin{array}{ll}\hat{G}_{11} & \hat{G}_{12} \\ \hat{G}_{21} & \hat{G}_{22}\end{array}\right)$ and then from the above every component $\hat{G}_{i j}$ has the factor $(1-\kappa)$. Therefore the singularity on $1-e^{-2 \pi i(C-B-A)}=0$. of $\hat{G}$ is removal. So $\hat{E}_{1}$ and $\hat{E}_{1}^{-1}$ are entire functions of $\alpha$ and $c$.

We have expressed $U_{\alpha}^{c}$ and $V_{\alpha}^{c}$ on the universal covering space $\tilde{\omega}_{r}$ of the base space $\omega_{r}$ with $U_{x}^{c}$ and $V_{\alpha}^{c}$ on the base space $\omega_{r}$, which we are to investigate. For this purpose to these functions we apply the connection formulae of the hyper geometric functions which are described bellow by the help of Kummer's transformations.

(C.F.)(1) $\quad F(A, B, C ; z)$

$$
\begin{aligned}
= & \frac{\Gamma(C) \Gamma(C-A-B)}{\Gamma(C-A) \Gamma(C-B)} F(A, B, A+B-C+1 ; 1-z) \\
& +\frac{\Gamma(A+B-C) \Gamma(C)}{\Gamma(A) \Gamma(B)}(1-z)^{C-B-A} \\
& \times F(C-A, C-B, C-A-B+1 ; 1-z) \text { in }|1-z|=\left|\frac{\eta}{\xi}\right|<1
\end{aligned}
$$

(C.F.)(2) $\quad F(A, B, C ; z)$

$$
\begin{aligned}
= & \frac{\Gamma(B-A) \Gamma(C)}{\Gamma(C-A) \Gamma(B)}(1-z)^{-A} F\left(A, C-B, A-B+1, \frac{1}{1-z}\right) \\
& +\frac{\Gamma(A-B) \Gamma(C)}{\Gamma(C-B) \Gamma(A)}(1-z)^{-B} \\
& \times F\left(B, C-A, B-A+1, \frac{1}{1-z}\right) \text { in }\left|\frac{1}{1-z}\right|=\left|\frac{\xi}{\eta}\right|<1
\end{aligned}
$$


(C.F.)(3) $\quad z^{1-c} F(A-C+1, B-C+1,2-C ; z)$

$$
\begin{aligned}
= & \frac{\Gamma(A+B-C) \Gamma(2-C)}{\Gamma(A-C+1) \Gamma(B-C+1)}(1-z)^{C-A-B} \\
& \times F(C-A, C-B, C-B-A+1 ; 1-z) \\
& +\frac{\Gamma(C-A-B) \Gamma(2-C)}{\Gamma(1-A) \Gamma(1-B)} F(A, B, A+B-C+1 ; 1-z) \\
& \quad \text { in }|1-z|=\left|\frac{\eta}{\xi}\right|<1
\end{aligned}
$$

(C.F.)(4) $\quad z^{1-c} F(A-C+1, B-C+1,2-C ; z)$

$$
\begin{aligned}
= & \frac{\Gamma(B-A) \Gamma(2-C)}{\Gamma(1-A) \Gamma(B-C+1)}(1-z)^{-A} e^{\pi i(C-1)} \\
& \times F\left(A, C-B, A-B+1 ; \frac{1}{1-z}\right) \\
& +\frac{\Gamma(A-B) \Gamma(2-C)}{\Gamma(1-B) \Gamma(A-C+1)}(1-z)^{-B} e^{\pi i(C-1)} \\
& \times F\left(B, C-A, B-A+1 ; \frac{1}{1-z}\right) \text { in }\left|\frac{1}{1-z}\right|=\left|\frac{\xi}{\eta}\right|<1
\end{aligned}
$$

Using these formulae we can list up the representations of $U_{\alpha}^{c}, \tilde{U}_{\alpha}^{c}, V_{\alpha}^{c}$ and $\tilde{V}_{\alpha}^{c}$ in $\left|\frac{\eta}{\xi}\right|<1$ and $\left|\frac{\xi}{\eta}\right|<1$ respectively.

We put $n^{*}=\frac{n}{2(n+1)}$ and $\alpha^{*}=\alpha+n^{*}, n^{* *}=1-n^{*}$ and $\alpha^{* *}=\alpha+n^{* *}$

$$
\begin{aligned}
& F_{1}\left(\alpha^{*}, c, D, n^{*} ; \zeta\right)=F\left(n^{*}-\alpha^{*}, n^{*}+\frac{\alpha^{*}+c-1}{D},-\alpha^{*}+\frac{\alpha^{*}+c-1}{D}+1 ; \zeta\right), \\
& F_{2}\left(\alpha^{*}, c, D, n^{*} ; \zeta\right)=F\left(n^{*}+\alpha^{*}, n^{*}+\frac{\alpha^{*}+c-1}{D}, \alpha^{*}+\frac{\alpha^{*}+c-1}{D}+1 ; \zeta\right), \\
& \gamma_{1}\left(\alpha^{*}, c, D, n^{*} ; \zeta\right)=\frac{\Gamma\left(2 n^{*}\right) \Gamma\left(\alpha^{*}+\frac{\alpha^{*}+c-1}{D}\right)}{\Gamma\left(n^{*}+\alpha^{*}\right) \Gamma\left(n^{*}+\frac{\alpha^{*}+c-1}{D}\right)} \frac{1}{\Gamma(\alpha+1)}, \\
& \gamma_{2}\left(\alpha^{*}, c, D, n^{*} ; \zeta\right)=\frac{\Gamma\left(2 n^{*}\right) \Gamma\left(-\alpha^{*}+\frac{\alpha^{*}+c-1}{D}\right)}{\Gamma\left(n^{*}-\alpha^{*}\right) \Gamma\left(n^{*}+\frac{\alpha^{*}+c-1}{D}\right)} \frac{1}{\Gamma(\alpha+1)} .
\end{aligned}
$$

We can show the following list of the representations of $U_{\alpha}^{c}, \tilde{U}_{\alpha}^{c}, V_{\alpha}^{c}$ and $\tilde{V}_{\alpha}^{c}$ 
in $\left|\frac{\eta}{\xi}\right|<1$ and $\left|\frac{\xi}{\eta}\right|<1$ respectively, by which we can know the behaviours, singularities and ramifications of $U_{\alpha}^{c}, \tilde{U}_{\alpha}^{c}, V_{\alpha}^{c}$ and $\tilde{V}_{\alpha}^{c}$ on the whole base space (F.B.) $\left(U_{\alpha}^{c}, \tilde{U}_{\alpha}^{c}, V_{\alpha}^{c}, \tilde{V}_{\alpha}^{c}\right.$ on the whole base space)

$$
\begin{aligned}
& U_{\alpha}^{c}(t, x)=\gamma_{1}\left(\alpha^{*}, c, D, n^{*}\right) \eta^{\alpha} F_{1}\left(\alpha^{*}, c,-D, n^{*} ; \frac{\xi}{\eta}\right) \\
& +\gamma_{2}\left(\alpha^{*}, c,-D, n^{*}\right) \eta^{-n^{*}-\left(\alpha^{*}+c-1\right) / D} F_{2}\left(\alpha^{*}, c, D, n^{*} ; \frac{\xi}{\eta}\right) \xi^{n^{*}+\left(\alpha^{*}+c-1\right) / D} \xi^{\alpha} \\
& \text { in }\left|\frac{\xi}{\eta}\right|<1 \\
& =\gamma_{1}\left(\alpha^{*}, c,-D, n^{*}\right) \xi^{\alpha} F_{1}\left(\alpha^{*}, c, D, n^{*} ; \frac{\eta}{\xi}\right) \\
& +\gamma_{2}\left(\alpha^{*}, c, D, n^{*}\right) \xi^{-n^{*}+\left(\alpha^{*}+c-1\right) / D} F_{2}\left(\alpha^{*}, c,-D, n^{*} ; \frac{\eta}{\xi}\right) \eta^{n^{*}-\left(\alpha^{*}+c-1\right) / D} \eta^{\alpha} \\
& \text { in }\left|\frac{\eta}{\xi}\right|<1 \\
& \tilde{U}_{\alpha}^{c}(t, x)=\gamma_{1}\left(\alpha^{*}, c, D, n^{* *}\right) e^{\pi i\left(n^{*}-n^{* *}\right)} \eta^{\alpha} F_{1}\left(\alpha^{*}, c,-D, n^{*} ; \frac{\xi}{\eta}\right) \\
& +\gamma_{2}\left(\alpha^{*}, c,-D, n^{* *}\right) e^{\pi i\left(n^{*}-n^{* *}\right)} \eta^{-n^{*}-\left(\alpha^{*}+c-1\right) / D} \\
& \times F_{2}\left(\alpha^{*}, c, D, n^{*} ; \frac{\xi}{\eta}\right) \xi^{n^{*}+\left(\alpha^{*}+c-1\right) / D} \xi^{\alpha} \quad \text { in }\left|\frac{\xi}{\eta}\right|<1 \\
& =\gamma_{1}\left(\alpha^{*}, c,-D, n^{* *}\right) \xi^{\alpha} F_{1}\left(\alpha^{*}, c, D, n^{*} ; \frac{\eta}{\xi}\right) \\
& +\gamma_{2}\left(\alpha^{*}, c, D, n^{* *}\right) \xi^{-n^{*}+\left(\alpha^{*}+c-1\right) / D} F_{2}\left(\alpha^{*}, c,-D, n^{*} ; \frac{\eta}{\xi}\right) \eta^{n^{*}-\left(\alpha^{*}+c-1\right) / D} \eta^{\alpha} \\
& \text { in }\left|\frac{\eta}{\xi}\right|<1 \\
& V_{\alpha}^{c}(t, x)=\gamma_{1}\left(\alpha^{* *}, c, D, n^{* *}\right) t \eta^{\alpha} F_{1}\left(\alpha^{* *}, c,-D, n^{* *} ; \frac{\xi}{\eta}\right) \\
& +\gamma_{2}\left(\alpha^{* *}, c,-D, n^{* *}\right) t \eta^{-n^{* *}-\left(\alpha^{* *}+c-1\right) / D} F_{2}\left(\alpha^{* *}, c, D, n^{* *} ; \frac{\xi}{\eta}\right) \xi^{\eta^{* *}+\left(\alpha^{* *}+c-1\right) / D} \xi^{\alpha} \\
& \text { in }\left|\frac{\xi}{\eta}\right|<1
\end{aligned}
$$




$$
\begin{aligned}
= & \gamma_{1}\left(\alpha^{* *}, c,-D, n^{* *}\right) t \xi^{\alpha} F_{1}\left(\alpha^{* *}, c, D, n^{* *} ; \frac{\eta}{\xi}\right) \\
& +\gamma_{2}\left(\alpha^{* *}, c, D, n^{* *}\right) t \xi^{-n^{* *}+\left(\alpha^{* *}+c-1\right) / D} F_{2}\left(\alpha^{* *}, c,-D, n^{* *} ; \frac{\eta}{\xi}\right) \eta^{n^{* *}-\left(\alpha^{* *}+c-1\right) / D} \eta^{\alpha} \\
& \text { in }\left|\frac{\eta}{\xi}\right|<1 \\
\tilde{V}_{\alpha}^{c}(t, x)= & \gamma_{1}\left(\alpha^{* *}, c, D, n^{*}\right) e^{\pi i\left(n^{* *}-n^{*}\right)} t \eta^{\alpha} F_{1}\left(\alpha^{* *}, c,-D, n^{* *} ; \frac{\xi}{\eta}\right) \\
& +\gamma_{2}\left(\alpha^{* *}, c,-D, n^{*}\right) e^{\pi i\left(n^{* *}-n^{*}\right)} t \eta^{-n^{* *}-\left(\alpha^{* *}+c-1\right) / D} \\
& \times F_{2}\left(\alpha^{* *}, c, D, n^{* *} ; \frac{\xi}{\eta}\right) \xi^{n^{* *}+\left(\alpha^{* *}+c-1\right) / D} \xi^{\alpha} \quad \text { in }\left|\frac{\xi}{\eta}\right|<1 \\
= & \gamma_{1}\left(\alpha^{* *}, c,-D, n^{*}\right) t \xi^{\alpha} F_{1}\left(\alpha^{* *}, c, D, n^{* *} ; \frac{\eta}{\xi}\right) \\
& +\gamma_{2}\left(\alpha^{* *}, c, D, n^{*}\right) t \xi^{-n^{* *}+\left(\alpha^{* *}+c-1\right) / D} F_{2}\left(\alpha^{* *}, c,-D, n^{* *} ; \frac{\eta}{\xi}\right) \eta^{n^{* *}-\left(\alpha^{* *}+c-1\right) / D} \eta^{\alpha} \\
& \text { in }\left|\frac{\eta}{\xi}\right|<1
\end{aligned}
$$

Thus we have seen the behaviours of $U_{\alpha}^{c}, \tilde{U}_{\alpha}^{c}, V_{\alpha}^{c}$ and $\tilde{V}_{\alpha}^{c}$ on the whole base space. For the proof the convergence of the formal solution, we need the estimates of these functions on the universal covering space, which are reduced to the estimates of these functions on the base space. Besides by (F.B.) those are reduced to the estimates of the hypergeometric functions $F_{1}\left(\alpha^{*}, c, \pm D, n^{*} ; \zeta\right)$, $F_{2}\left(\alpha^{*}, c, \pm D, n^{*} ; \zeta\right), \quad F_{1}\left(\alpha^{* *}, c, \pm D, n^{* *} ; \zeta\right)$ and $F_{2}\left(\alpha^{* *}, c, \pm D, n^{* *} ; \zeta\right)$. Using following lemmata, we estimate these hypergeometric functions.

Lemma (E.H.) If $A, B, C$ and $C-B-A$ are positive, we have the next estimate

$$
|F(A, B, C ; \zeta)| \leq F(A, B, C, 1)=\frac{\Gamma(C) \Gamma(C-B-A)}{\Gamma(C-A) \Gamma(C-B)} \quad \text { in }|\zeta|<1 .
$$

Lemma (M.H.) (1) if $A, B, C$ are complex numbers such that $\delta=C-B-A$ is real and nonnegative, and $\operatorname{Re} A$ and $\operatorname{Re} B$ are positive, we have the next majoration relations for an adequate positive constant $\varepsilon$

$$
F(A, B, C ; \zeta) \ll F\left(|A|_{\varepsilon},|B|_{\varepsilon},|A|_{\varepsilon}+|B|_{\varepsilon}+\delta ; \zeta\right) \quad \text { in }|\zeta|<1 .
$$

where $|z|_{\varepsilon}=|\operatorname{Re} z|+\varepsilon|\operatorname{Im} z|$. 
(2) if $A, B, C$ are complex numbers such that $\delta=C-B-A$ is real and nonnegative, we have the next majoration relation for positive constants $T$ and $\varepsilon$

$$
F(A, B, C ; \zeta) \ll T^{\beta+1} F\left(|A|_{\varepsilon},|B|_{\varepsilon},|A|_{\varepsilon}+|B|_{\varepsilon}+\delta ; \zeta\right) \quad \text { in }|\zeta|<1
$$

where $\beta=\operatorname{int}(\max (0,-\operatorname{Re} A,-\operatorname{Re} B,-\operatorname{Re}(A+B+\delta)))$.

First applying this lemma (M.H.) to $F_{1}\left(\alpha^{*}, c, \pm D, n^{*} ; \zeta\right), F_{2}\left(\alpha^{*}, c, \pm D, n^{*} ; \zeta\right)$, $F_{1}\left(\alpha^{* *}, c, \pm D, n^{* *} ; \zeta\right)$ and $F_{2}\left(\alpha^{* *}, c, \pm D, n^{* *} ; \zeta\right)$ we have following majoration estimates in the unit disk $|\zeta|<1$. We denote a suitable positive constant by $C$ in bellow;

$$
\begin{aligned}
& F_{1}\left(\alpha^{*}, c, \pm D, n^{*} ; \zeta\right) \ll C T^{\beta \pm} F\left(\left|n^{*}-\alpha^{*}\right|_{\varepsilon},\left|n^{*} \pm \frac{\alpha^{*}+c-1}{D}\right|_{\varepsilon},\left|n^{*}-\alpha^{*}\right|_{\varepsilon}\right. \\
& \left.+\left|n^{*} \pm \frac{\alpha^{*}+c-1}{D}\right|_{\varepsilon}+1-2 n^{*} ; 1\right) \quad \text { where } \\
& \beta \pm=\operatorname{int}\left(\operatorname { m a x } \left(-\operatorname{Re}\left(n^{*}-\alpha^{*}\right),-\operatorname{Re}\left(n^{*} \pm \frac{\alpha^{*}+c-1}{D}\right),\right.\right. \\
& \left.\left.-\operatorname{Re}\left(-\alpha^{*} \pm \frac{\alpha^{*}+c-1}{D}+1\right)\right)\right) \\
& F_{2}\left(\alpha^{*}, c, \pm D, n^{*} ; \zeta\right) \ll C T^{\beta \pm} F\left(\left|n^{*}+\alpha^{*}\right|_{\varepsilon},\left|n^{*} \pm \frac{\alpha^{*}+c-1}{D}\right|_{\varepsilon}\right. \\
& \left.\left|n^{*}+\alpha^{*}\right|_{\varepsilon}+\left|n^{*} \pm \frac{\alpha^{*}+c-1}{D}\right|_{\varepsilon}+1-2 n^{*} ; 1\right) \text { where } \\
& \beta \pm=\operatorname{int}\left(\operatorname { m a x } \left(-\operatorname{Re}\left(n^{* *}+\alpha^{* *}\right),-\operatorname{Re}\left(n^{* *} \pm \frac{\alpha^{* *}+c-1}{D}\right),\right.\right. \\
& \left.\left.\operatorname{Re}\left(\alpha^{* *} \pm \frac{\alpha^{* *}+c-1}{D}+1\right)\right)\right) \\
& F_{1}\left(\alpha^{* *}, c, \pm D, n^{* *} ; \zeta\right) \ll C T^{\beta \pm} F\left(\left|\alpha^{* *}-n^{* *}\right|_{\varepsilon},\left|n^{* *} \pm \frac{\alpha^{* *}+c-1}{D}\right|_{\varepsilon}\right. \\
& \left.\left|\alpha^{* *}-n^{* *}\right|_{\varepsilon}+\left|n^{* *} \pm \frac{\alpha^{* *}+c-1}{D}\right|_{\varepsilon}+1-2 n^{* *} ; 1\right) \text { where } \\
& \beta \pm=\operatorname{int}\left(\operatorname { m a x } \left(-\operatorname{Re}\left(n^{* *}-\alpha^{* *}\right),-\operatorname{Re}\left(n^{* *} \pm \frac{\alpha^{* *}+c-1}{D}\right)\right.\right. \\
& \left.\left.-\operatorname{Re}\left(-\alpha^{* *} \pm \frac{\alpha^{* *}+c-1}{D}+1\right)\right)\right)
\end{aligned}
$$




$$
\begin{aligned}
& F_{2}\left(\alpha^{* *}, c, \pm D, n^{* *} ; \zeta\right) \ll C T^{\beta \pm} F\left(\left|\alpha^{* *}+n^{* *}\right|_{\varepsilon},\left|n^{* *} \pm \frac{\alpha^{* *}+c-1}{D}\right|_{\varepsilon},\right. \\
& \left.\left|\alpha^{* *}+n^{* *}\right|_{\varepsilon}+\left|n^{* *} \pm \frac{\alpha^{* *}+c-1}{D}\right|_{\varepsilon}+1-2 n^{* *} ; 1\right) \text { where } \\
& \beta \pm=\operatorname{int}\left(\operatorname { m a x } \left(-\operatorname{Re}\left(n^{* *}+\alpha^{* *}\right),-\operatorname{Re}\left(n^{* *} \pm \frac{\alpha^{* *}+c-1}{D}\right)\right.\right. \\
& \left.\left.-\operatorname{Re}\left(\alpha^{* *} \pm \frac{\alpha^{* *}+c-1}{D}+1\right)\right)\right) .
\end{aligned}
$$

Secondly we apply lemma (E.H.) to these hypergeometric functions appearing in the right hand sides above majoration relations. then we have next estimates of these functions in the unit disk $|\zeta| \leq 1$;

$$
\begin{gathered}
F_{1}\left(\alpha^{*}, c, \pm D, n^{*} ; \zeta\right) \\
\leq C T^{\beta \pm} \frac{\Gamma\left(\left|n^{*}-\alpha^{*}\right|_{\varepsilon}+\left|n^{*} \pm \frac{\alpha^{*}+c-1}{D}\right|_{\varepsilon}+1-2 n^{*}\right) \Gamma\left(1-2 n^{*}\right)}{\Gamma\left(\left|n^{*} \pm \frac{\alpha^{*}+c-1}{D}\right|_{\varepsilon}+1-2 n^{*}\right) \Gamma\left(\left|n^{*}-\alpha^{*}\right|_{\varepsilon}+1-2 n^{*}\right)} \text { where } \\
\beta \pm=\operatorname{int}\left(\operatorname { m a x } \left(-\operatorname{Re}\left(n^{*}-\alpha^{*}\right),-\operatorname{Re}\left(n^{*} \pm \frac{\alpha^{*}+c-1}{D}\right)\right.\right. \\
\left.\left.-\operatorname{Re}\left(-\alpha^{*} \pm \frac{\alpha^{*}+c-1}{D}+1\right)\right)\right)
\end{gathered}
$$

$F_{2}\left(\alpha^{*}, c, \pm D, n^{*} ; \zeta\right)$

$$
\begin{gathered}
\leq C T^{\beta \pm} \frac{\Gamma\left(\left|n^{*}+\alpha^{*}\right|_{\varepsilon}+\left|n^{*} \pm \frac{\alpha^{*}+c-1}{D}\right|_{\varepsilon}+1-2 n^{*}\right) \Gamma\left(1-2 n^{*}\right)}{\Gamma\left(\left|n^{*} \pm \frac{\alpha^{*}+c-1}{D}\right|_{\varepsilon}+1-2 n^{*}\right) \Gamma\left(\left|n^{*}+\alpha^{*}\right|_{\varepsilon}+1-2 n^{*}\right)} \text { where } \\
\beta \pm=\operatorname{int}\left(\operatorname { m a x } \left(-\operatorname{Re}\left(n^{* *}+\alpha^{* *}\right),-\operatorname{Re}\left(n^{* *} \pm \frac{\alpha^{* *}+c-1}{D}\right)\right.\right. \\
\left.\left.\operatorname{Re}\left(\alpha^{* *} \pm \frac{\alpha^{* *}+c-1}{D}+1\right)\right)\right),
\end{gathered}
$$

$F_{1}\left(\chi^{* *}, c, \pm D, n^{* *} ; \zeta\right)$

$$
\leq C T^{\beta \pm} \frac{\Gamma\left(\left|\alpha^{* *}-n^{* *}\right|_{\varepsilon}+\left|n^{* *} \pm \frac{\alpha^{* *}+c-1}{D}\right|_{\varepsilon}+1-2 n^{* *}\right) \Gamma\left(1-2 n^{* *}\right)}{\Gamma\left(\left|n^{* *} \pm \frac{\alpha^{* *}+c-1}{D}\right|_{\varepsilon}+1-2 n^{* *}\right) \Gamma\left(\left|\alpha^{* *}-n^{* *}\right|_{\varepsilon}+1-2 n^{* *}\right)} \text { where }
$$




$$
\begin{gathered}
\beta \pm=\operatorname{int}\left(\operatorname { m a x } \left(-\operatorname{Re}\left(n^{* *}+\alpha^{* *}\right),-\operatorname{Re}\left(n^{* *} \pm \frac{\alpha^{* *}+c-1}{D}\right),\right.\right. \\
\left.\left.\operatorname{Re}\left(\alpha^{* *} \pm \frac{\alpha^{* *}+c-1}{D}+1\right)\right)\right)
\end{gathered}
$$

$F_{2}\left(\alpha^{* *}, c, \pm D, n^{* *} ; \zeta\right)$

$$
\begin{gathered}
\leq C T^{\beta \pm} \frac{\Gamma\left(\left|\alpha^{* *}+n^{* *}\right|_{\varepsilon}+\left|n^{* *} \pm \frac{\alpha^{* *}+c-1}{D}\right|_{\varepsilon}+1-2 n^{* *}\right) \Gamma\left(1-2 n^{* *}\right)}{\Gamma\left(\left|n^{* *} \pm \frac{\alpha^{* *}+c-1}{D}\right|_{\varepsilon}+1-2 n^{* *}\right) \Gamma\left(\left|\alpha^{* *}+n^{* *}\right|_{\varepsilon}+1-2 n^{* *}\right)} \text { where } \\
\beta \pm=\operatorname{int}\left(\operatorname { m a x } \left(-\operatorname{Re}\left(n^{* *}+\alpha^{* *}\right),-\operatorname{Re}\left(n^{* *} \pm \frac{\alpha^{* *}+c-1}{D}\right)\right.\right. \\
\left.\left.-\operatorname{Re}\left(\alpha^{* *} \pm \frac{\alpha^{* *}+c-1}{D}+1\right)\right)\right) .
\end{gathered}
$$

We remark that in the above estimate of $F_{1}\left(\alpha^{*}, c, \pm D, n^{*} ; \zeta\right)$, $F_{2}\left(\alpha^{*}, c, \pm D, n^{*} ; \zeta\right), \quad F_{1}\left(\alpha^{* *}, c, \pm D, n^{* *} ; \zeta\right) \quad F_{2}\left(\alpha^{* *}, c, \pm D, n^{* *} ; \zeta\right)$ in the unit disk $|\zeta| \leq 1$ of the base space and $\gamma_{1}\left(\alpha^{*}, c, D, n^{*} ; \zeta\right), \gamma_{2}\left(\alpha^{*}, c, D, n^{*} ; \zeta\right)$ etc., we find $\Gamma$ factors in the similar form $\frac{\Gamma\left(A_{1}\right) \Gamma\left(A_{2}\right)}{\Gamma\left(B_{1}\right) \Gamma\left(B_{2}\right)}$ such as $A_{1}+A_{2}=B_{1}+B_{2}$. Using these estimates we obtain the following estimates of $U_{\alpha+r-1}^{c-k+1}(t, x), \tilde{U}_{\alpha+r-1}^{c-k+1}(t, x)$, $V_{\alpha+r-1}^{c-k+1}(t, x)$ and $\tilde{V}_{\alpha+r-1}^{c-k+1}(t, x)$ on the base space $\omega_{\lambda}=\{(t, x): 0 \neq|\xi|<\lambda, 0 \neq|\eta|<$ $\lambda\}$.

There exists positive constants $C(\alpha, c)$ and $T_{1}, T_{2}$ independent of $r, k$ such that $\left|U_{\alpha+r-1}^{c-k+1}(t, x)\right|,\left|\tilde{U}_{\alpha+r-1}^{c-k+1}(t, x)\right|,\left|V_{\alpha+r-1}^{c-k+1}(t, x)\right|,\left|\tilde{V}_{\alpha+r-1}^{c-k+1}(t, x)\right| \leq C(\alpha, c) T_{1}^{r} T_{2}^{k} \frac{\lambda^{r-k}}{r !}$ in $\omega_{\lambda}$.

Let $\tilde{K}$ be any compact set in the universal covering space $\tilde{\omega}_{\lambda}$ over $\omega_{\lambda}$. Let $l$ be any loop in $\tilde{\omega}_{\lambda}$. If $l$ encircles $\xi=0$ or $\eta=0 \beta$ times in the positive or negative sense, we must estimate $g_{1}^{\beta} . \quad g_{1}=\hat{D}^{-1} \hat{C}^{-1}\left(\begin{array}{cc}1 & 0 \\ 0 & e^{2 \pi i(C-B-A)}\end{array}\right) \hat{C} \hat{D}$, so we must
estimate $e^{2 \pi i \beta(C-B-A)}$ where

$$
\begin{aligned}
& C-B-A=\alpha^{*}+r-1-\frac{1}{D}\left(\alpha^{*}+c+(r-k)-1\right) \text { for } U_{\alpha+r-1}^{c-k+1} \text { and } \tilde{U}_{\alpha+r-1}^{c-k+1}, \\
& C-B-A=\alpha^{* *}+r-1-\frac{1}{D}\left(\alpha^{* *}+c+(r-k)-1\right) \text { for } V_{\alpha+r-1}^{c-k+1} \text { and } \tilde{V}_{\alpha+r-1}^{c-k+1}
\end{aligned}
$$

that is $\exp \left(\left(\operatorname{Re}\left(\frac{2 \pi i}{D}\right)\right) \beta(r-k)\right) . \quad$ Because $\exp \left(\left(\operatorname{Re}\left(\frac{2 \pi i}{D}\right)\right) \beta(r-k)\right)<$ $C(\tilde{K})(r-k)$ ! where $C$ is a constant independent of $r-k$, we have the following estimates of $U_{\alpha+r-1}^{c-k+1}(t, x), \quad \tilde{U}_{\alpha+r-1}^{c-k+1}(t, x), \quad V_{\alpha+r-1}^{c-k+1}(t, x)$ and $\tilde{V}_{\alpha+r-1}^{c-k+1}(t, x)$ on any compact set $\tilde{K}$ in the universal covering space $\tilde{\omega}_{\lambda}$ over $\omega_{\lambda}$. 
Proposition (E.U.) There exist positive constants $T_{1}, T_{2}, C(\alpha, c, \tilde{K})$ independent of $r, k$ such that

$$
\begin{gathered}
\left|U_{\alpha+r-1}^{c-k+1}(l(P))\right|,\left|\tilde{U}_{\alpha+r-1}^{c-k+1}(l(P))\right|,\left|V_{\alpha+r-1}^{c-k+1}(l(P))\right|, \\
\left|\tilde{V}_{\alpha+r-1}^{c-k+1}(l(P))\right| \leq C(\alpha, c, \tilde{K}) T_{1}^{r} T_{2}^{k} \frac{(r-k) !}{r !} \lambda^{r}
\end{gathered}
$$

on any compact set $\tilde{K}$ in the universal covering space $\tilde{\omega}_{\lambda}$ over $\omega_{\lambda}$.

Example. We consider the next simple Cauchy problem

$$
\left(P_{c}-d t^{n-1}\right) u(t, x)=0
$$

with the initial data $u(0, x)=f_{\alpha}(x)$ and $u_{t}(0, x)=0$

where $d$ is a constant.

This Cauchy problem has the explicit representation of the solution;

$$
u(t, x)=\sum_{r=0}^{\infty} \frac{d^{r}}{r !} \sum_{k=0}^{r}(-1)^{k}\left(\begin{array}{l}
r \\
k
\end{array}\right) U_{\alpha+r}^{c-k}(t, x) .
$$

To prove this fact it is enough to use the formula $P_{c}\left(U_{\alpha-1}^{c-k}\right)=-k t^{n-1} U_{\alpha-2}^{c-k+1}$

\section{DePartMent of ENGineEring DOSHISHA UNIVERSITY}

\section{References}

[G.S.1] V. Guillemin and D. Schaeffer, On a certain class of Fuchsian partial differential equations, Duke Math., 44 (1977), 157-199.

[H.L.W.1] Y. Hamada, J. Leray et C. Wagschal, Systèms d'équattion aux dérivées partielles a caractéristiques multiples; problème de Cauchy ramifié, hyperbolicité partielles, J. Math. pure et appl., 55 (1976), 297-352.

[H.N.1] Y. Hamada and G. Nakamura, On the singularities of the solution of the Cauchy problem for the operator with non uniform multiple characteristics, Annali della Scoula Norm. Sup. di Pisa, 4 (1977), 725-755.

[Ki.1] T. Kimura Hypergeometric functions of two variables, Minnesota Univ., (1973).

[Ko.1] T. Kobayashi, On the singular Cauchy problem for operators with variable involutive characteristics. J. Fac. Sci. Univ. Tokyo, 29 (1982), 97-142.

[L.1] P. E. Leichtnam Le problème de Cauchy ramifié, Ann. Sci, Ecole Norm. Sup., 23 (1990), 367-443.

[P.1] J. Persson, Ramification of the solutions of the Cauchy problem for a special second order equation with singular data, Comm, PDE., 17 (1992), 23-31.

[S.V.W.1] D. Shiltz, J. Vaillant et C. Wagschal, Problème de Cauchy ramifié: racine caractéristique double ou triple en involution, J. Math. pure et appl., 61 (1982), 423-443.

[S.S.1] B. Sternin \& V. Shatalov, Differential Equations on Complex Manifolds (MIA Vol.276), Kluwer Acad. Publ., (1994). 
[U.1] J. Urabe, Hamada's theorem for a certain type of the operators with double characteristics, J. Math. Kyoto Univ., 23 (1983).

[U.2] Meromorphic representations of the solutions of the Singular Cauchy problem I, J. Math. Kyoto Univ., 26 (1986).

[U.3] Meromorphic representations of the solutions of the Singular Cauchy problem II, J. Math. Kyoto Univ., 28 (1988). 